\title{
NORTH-FINDING WITH 0.004 RADIAN PRECISION USING A SILICON MEMS QUADRUPLE MASS GYROSCOPE WITH Q-FACTOR OF 1 MILLION
}

\author{
I.P. Prikhodko, A.A. Trusov, and A.M. Shkel
}

MicroSystems Laboratory, University of California, Irvine, USA

\begin{abstract}
We demonstrate north-finding capability with a measured 4 milliradian (mrad) $1-\sigma$ uncertainty using an in-house developed vibratory silicon MEMS quadruple mass gyroscope (QMG). We instrumented a vacuum packaged QMG with isotropic $Q$-factor of 1.2 million and bias instability of $0.1 \% \mathrm{hr}$ for azimuth detection by measuring the Earth's rotation. Continuous rotation ("carouseling") produced azimuth datapoints with uncertainty diminishing as the square root of the number of turns. Integration of 100 datapoints with normally distributed errors reduced uncertainty to $4 \mathrm{mrad}$, beyond the noise limit of the QMG. We also implemented selfcalibration methods, including temperature compensation and discreet $\pm 180^{\circ}$ turning ("maytagging") as potential alternatives to the carouseling.
\end{abstract}

\section{INTRODUCTION}

North-finding with single digit mrad precision is required for setting the initial orientation in the dead reckoning and targeting. Practical limitations of geodetic, celestial, magnetic, and GPS-based methods make high performance gyroscopes desired for true North finding ("gyrocompassing"). Although commercially available fiber optic, ring laser, and hemispherical resonator gyroscopes can be used for precision gyrocompassing, they are not perfectly suited for man-portable or handheld systems due to their size, weight and power (SWaP) characteristics. North-finding based on micromachined gyroscopes is an intriguing possibility, but silicon MEMS are yet to establish credibility in the high-precision domain [1]. Gyrocompassing requires repeatable and stable measurements of extremely low angular rates (fractions of the Earth's rate). For instance, a bias drift of only $1 \% / \mathrm{hr}$ leads to a $100 \mathrm{mrad}$ azimuth uncertainty at a $45^{\circ}$ latitude, which translates into a $9 \mathrm{~m}$ location error per each $100 \mathrm{~m}$ of dead reckoning or targeting.

Several approaches have been explored for the reduction of drift in MEMS gyroscopes, including carouseling [2,3] and maytagging [4]. Nevertheless, single digit mrad error is often assumed unattainable by MEMS technology. Our latest progress in the design, packaging and control electronics has enabled sub-degree per hour MEMS quadruple mass gyroscope (QMG) [3,5]. This paper reports the development and application of the high performance micromachined QMG for gyrocompassing.

\section{NORTH-FINDING TEST-BED}

The section describes a north-finding setup consisting of a QMG sensor, a tilt stage, and a rate table, Figure 1.

\section{Sensor and Electronics}

A stand-alone QMG [3] was fabricated using an inhouse SOI process and vacuum sealed using the ceramic

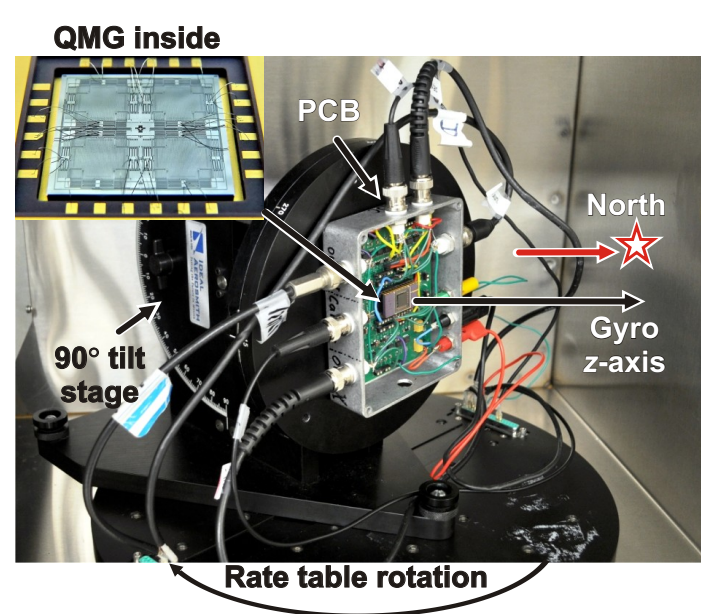

Figure 1: Photo of the north-finding setup, including a $Q M G$ rate sensor, a $90^{\circ}$ tilt stage, and a rate table.

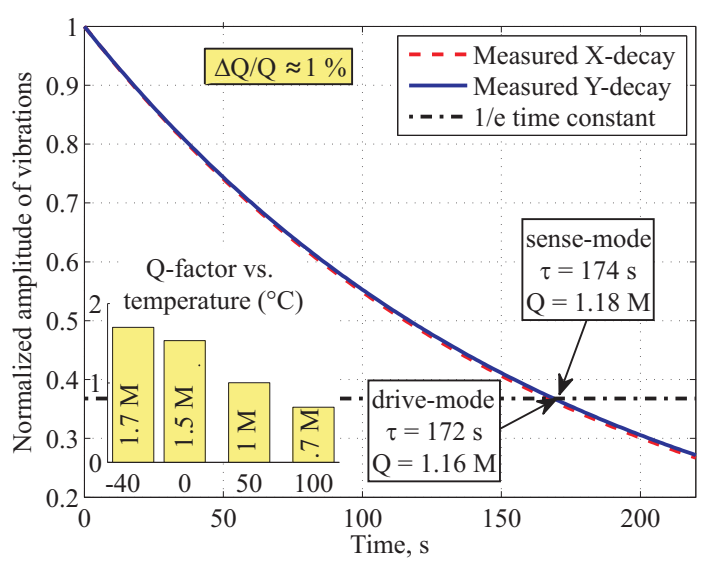

Figure 2: Experimental characterization of the vacuum sealed $Q M G$, revealing drive- and sense-mode $Q$-factor of 1.18 and 1.16 million. Inset: $Q$ vs. temperature.

package level technology. The QMG sensor was mounted on a PCB with front-end amplifiers and installed on the 1291 Ideal Aerosmith rate table enclosed in a thermal chamber. All reported experiments were carried out using a custom PCB connected to a HF2 digital lock-in amplifier from the Zurich Instruments (ZI), providing control and signal conditioning for the QMG [3].

For the angular rate measurements, the drive-mode was operated closed loop; the sense-mode remained open loop. A PLL drive loop sustained oscillation at resonance and provided reference for signals demodulation. An automatic gain control (AGC) stabilized the amplitude of drive motion. Rotation was detected by demodulating the sense-mode signal. The rate measurements were performed at a $0.16 \mathrm{~Hz}$ separation between the drive- and the sense-mode frequencies.

\section{Sensor Characterization}

The mechanical characterization of the QMG sensor with a $2 \mathrm{kHz}$ resonant frequency was performed by ring- 


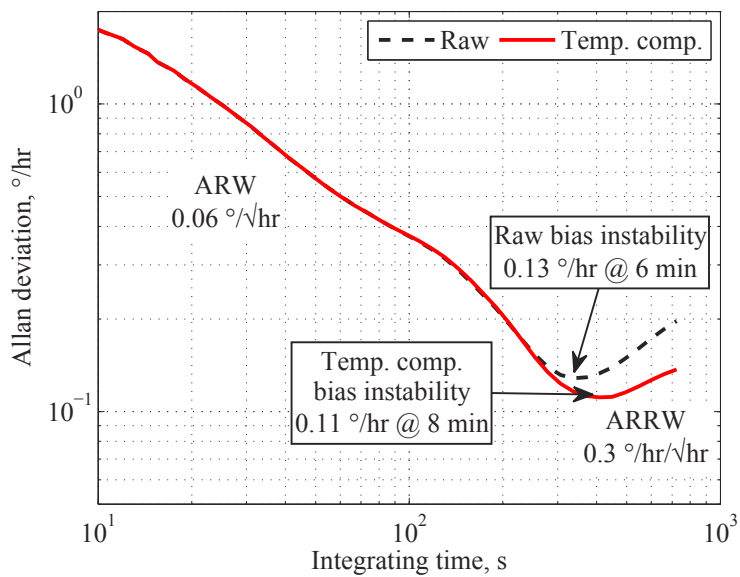

Figure 3: Measured Allan deviation of zero rate outputs, demonstrating a $0.06 \% \sqrt{h r}$ ARW and a $0.11 \% \mathrm{hr}$ bias instability for the temperature compensated data.

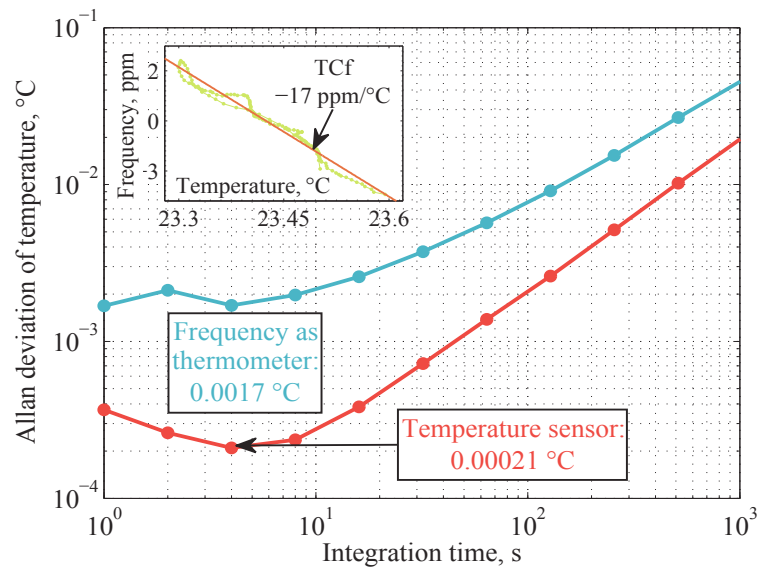

Figure 4: Allan deviation of reference temperature sensor and drive-mode frequency used as thermometer $\left(10^{-3}{ }^{\circ} \mathrm{C}\right.$ precision). Inset: temperature coefficient of frequency.

down tests. Figure 2 reveals drive- and sense-mode $Q$ factors of 1.17 million with $\Delta Q / Q$ of $1 \%$, which translates into the fundamental mechanical-thermal resolution limit of $10^{-4} \% / \mathrm{h} \mathrm{hr}$. Thermal cycling demonstrated $Q$-factors of 0.7 million for temperatures up to $100{ }^{\circ} \mathrm{C}$. The frequency symmetry evaluated in [5] confirmed closely matched temperature coefficients of frequency (TCF) in a wide temperature range with a $0.2 \mathrm{ppm} /{ }^{\circ} \mathrm{C}$ uncertainty.

The low dissipation QMG sensor demonstrated low noise characteristics. The Allan deviation plot of a zero rate output recorded for 1.5 hours is shown in Figure 3. For relatively short averaging time the QMG output is dominated by the angle random walk of $0.06 \% / \sqrt{\mathrm{hr}}$. The bias instability reaches a value of $0.13 \% / \mathrm{hr}$ for $6 \mathrm{~min}$ of averaging. For longer integration, a slope larger than $\tau^{+1 / 2}$ is observed. This indicates a temperature ramp, which is removed by the temperature self-compensation.

\section{Temperature Self-Compensation}

Most MEMS devices are sensitive to environmental fluctuations and require temperature compensation. For instance, high stability resonators employ secondary mode as a thermometer for self-compensation of primary mode drifts [6]. While the drive-mode of the QMG is controlled by PLL and AGC closed loops, the sense-mode mode is open loop and susceptible to temperature variations. The

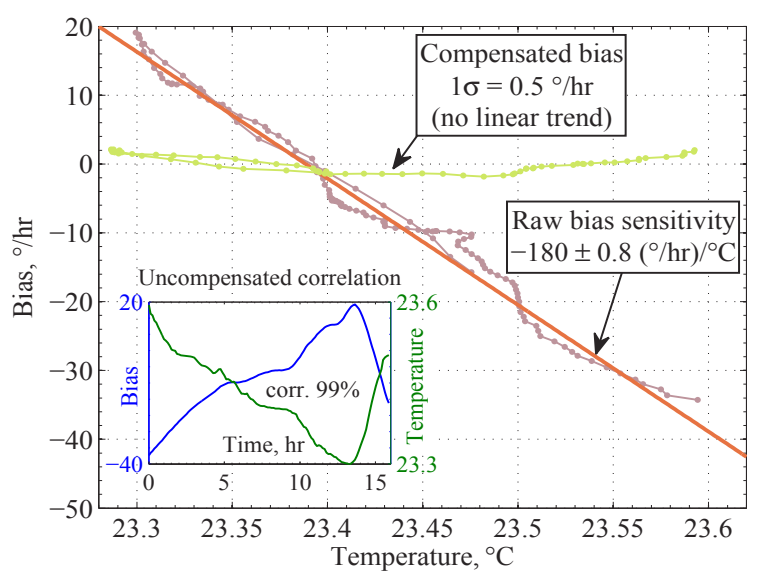

Figure 5: QMG thermal dependence. The uncompensated bias sensitivity is $-180\left({ }^{\circ} / \mathrm{hr}\right) /{ }^{\circ} \mathrm{C}$; self-calibration removes a linear trend. Inset: bias and temperature correlation.

uncompensated temperature sensitivity of $1000(\% / \mathrm{hr}) /{ }^{\circ} \mathrm{C}$ is customary for MEMS [7], and presents a challenge for the long-term measurements of the Earth's rate $\left(15^{\circ} / \mathrm{hr}\right)$.

Similarly to high stability resonators, we utilized the drive-mode frequency for a self-compensation of the gyro sense-mode drifts. A change in drive frequency (relative to the ovenized quartz reference) acted as an embedded thermometer free from thermal lag or hysteresis. The TCF of the QMG was determined using a $10^{-4}{ }^{\circ} \mathrm{C}$ resolution thermistor from GEC Instruments. The room temperature and frequency were recorded for $24 \mathrm{hr}$ and analyzed using the Allan deviation. The TCF value of $-17 \pm 0.1 \mathrm{ppm} /{ }^{\circ} \mathrm{C}$ calculated from the linear fit was applied for the frequency to temperature conversion, Figure 4. Owing to high- $Q$ factor, the measured frequency stability of 0.03 ppm provided a temperature precision of $0.002{ }^{\circ} \mathrm{C}$, on par with state-of-the-art MEMS resonant thermometers [8].

The temperature self-compensation using the drivemode frequency as thermometer improved the QMG bias stability to $0.11 \% \mathrm{hr}$, removed the temperature ramp, and resolved the rate random walk of $0.3 \% / \mathrm{hr} / \sqrt{\mathrm{hr}}$, Figure 3 . Most importantly, this method removed a $-180(\% / \mathrm{hr}) /{ }^{\circ} \mathrm{C}$ linear sensitivity of the null offset (bias) to below $0.5 \% \mathrm{hr}$ uncertainty, Figure 5, attesting feasibility of the long-term stable measurements required for the north-finding.

\section{NORTH-FINDING BY MAYTAGGING}

This section presents 2-point gyrocompassing results.

\section{Basic Principles}

The true North orientation (as opposed to the magnetic North) is found by observing the horizontal (tangential) component of the Earth's rotation vector. Since MEMS gyroscopes may exhibit long-term drift (not induced by temperature), a bias compensation is required for north-finding. A 2-point gyrocompassing [4] mitigates additive bias errors through differential azimuth measurement. The azimuth detection is accomplished by the $\pm 180^{\circ}$ turning (maytagging) of the gyroscope sensitive axis. The outputs of the gyroscope aligned to a local vertical level during maytagging are:

$$
\begin{gathered}
\omega(0)=\Omega_{E} \cos \alpha+b, \\
\omega(180)=-\Omega_{E} \cos \alpha+b,
\end{gathered}
$$




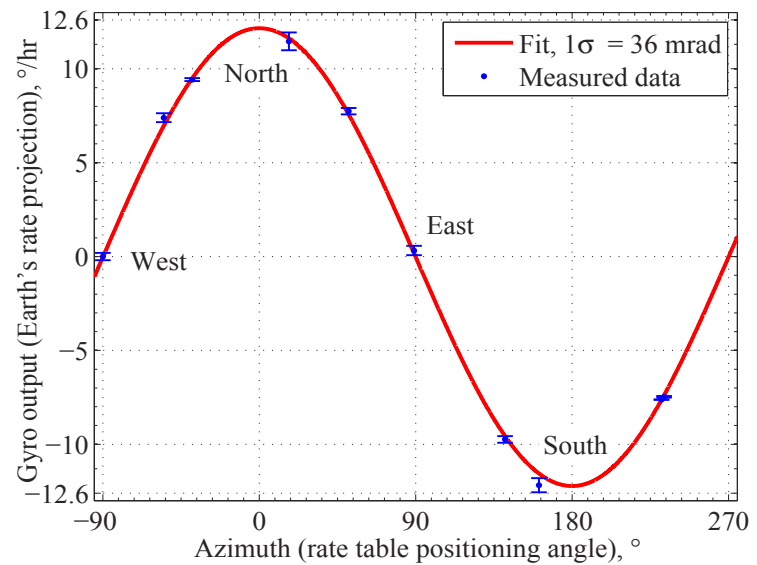

Figure 6: Measured projection of Earth' rate as function of azimuth using the differential maytagging approach. Gyro output is $12.6^{\circ} \mathrm{hr}$ (Irvine, CA) if pointing North.

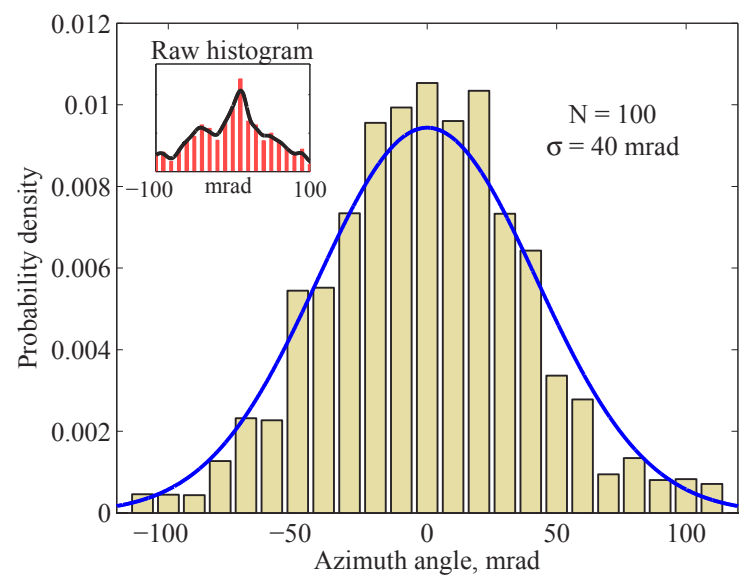

Figure 7: Azimuth histogram with normal distribution fit after temperature self-compensation, showing a $40 \mathrm{mrad}$ error of East-West maytagging. Inset: raw histogram.

where $\Omega_{E}$ is the Earth's rotation rate at a given latitude, $\alpha$ is the azimuth angle (heading angle), $b$ is the gyroscope bias (null offset). The azimuth and bias are recovered by differencing or summing outputs (1)-(2). The method also algorithmically cancels out acceleration-induced errors due to misalignment of the gyroscope with the gravity.

\section{Error Propagation}

The maytagging approach assumes constant bias during the 2-point gyrocompassing. The uncompensated bias $\delta b$ propagates to a differential azimuth measurement:

$$
\omega(0)-\omega(180)=2 \Omega_{E} \cos \alpha+\delta b,
$$

and corrupts calculation of the true azimuth value $\alpha$ :

$$
\hat{\alpha}=\arccos \left(\cos \alpha+\delta b / 2 \Omega_{E}\right) \approx \alpha-\delta b /\left(2 \Omega_{E}|\sin \alpha|\right) \text {. }
$$

The equation suggests error reduction for angles $\alpha= \pm 90^{\circ}$. In other words, best precision is achieved by maytagging in the East-West direction (as opposed to North-South).

\section{Experimental Results}

The North direction was detected by changing the orientation of the gyroscope $z$-axis relative to the Earth's rotation vector. The horizontal component was observed after orienting the QMG sensitive axis parallel to the local vertical plane, as shown in Figure 1. A rate table was used to position the gyroscope in the local horizontal plane.

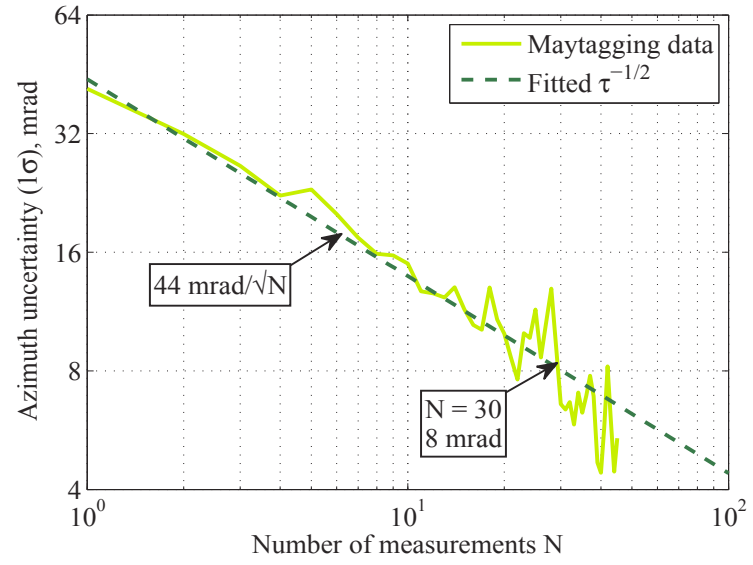

Figure 8: Azimuth error (1-) as a function of number of filtered measurements using Fig. 7 data. Error scales down as $44 \mathrm{mrad} / \sqrt{ } \mathrm{N}$, resulting in 8 mrad after $N=30$.

Figure 6 shows the horizontal component of Earth's vector as a function of positioning angle. Each datapoint is produced by a two position differential measurement of the QMG output according to (3). The measurement time was determined by the time necessary to reach the bias instability. According to Figure 3, the minimum Allan deviation of $0.11^{\circ} / \mathrm{hr}$ occurs after $8 \mathrm{~min}$, allowing for 3-4 min averaging time at each position. The phase of the sinusoidal fit curve with 36 mrad residual error was used to determine the North direction. The sinusoid amplitude was $12.6^{\circ} / \mathrm{hr}$, in good agreement with the Earth's rate component at $33.4^{\circ} \mathrm{N}$ latitude (Irvine, CA).

The maytagging precision was measured by statistical analysis. The classical estimation of azimuth error $\sigma(\alpha)$

$$
\sigma(\alpha)=\arctan \left(-b / \Omega_{E}\right)
$$

suggested a $0.01 \% \mathrm{hr}$ bias instability requirement to achieve $1 \mathrm{mrad}$ precision. The same uncertainty can be reached by filtration of multiple azimuth datapoints. The filtering however requires a normal distribution of errors and increases the north-finding time. To evaluate the distribution of noise we collected up to 100 multiple-turn datapoints. The East-West direction was chosen to minimize error propagation. Azimuth probability density histograms of raw and temperature self-compensated data is shown in Figure 7. The fit to normal distribution curve revealed a Gaussian error model for compensated data. Filtration diminished uncertainty with a $44 \mathrm{mrad} / \sqrt{ } N$ slope, providing a $8 \mathrm{mrad}$ precision after $N=30$ averages, Figure 8 .

\section{NORTH-FINDING BY CAROUSELING}

In this section we evaluate the carouseling approach for the bias compensated north-finding [2]. In contrast to the discreet maytagging, the carouseling requires continuous rotation of the gyroscope sensitive axis. The technique relies on modulation of the constant Earth's rate to separate bias, scale factor, and temperature errors.

The QMG sensor mounted on a rate table was rotated in local horizontal plane with $1 \%$ sate. This resulted in periodic rotation of the QMG sensitive axis and modulation of the Earth's constant rate with a $6 \mathrm{~min}$ period. Figure 9 shows a QMG output from a carouseling run. As expected, the sinusoidal variation was maximum, 


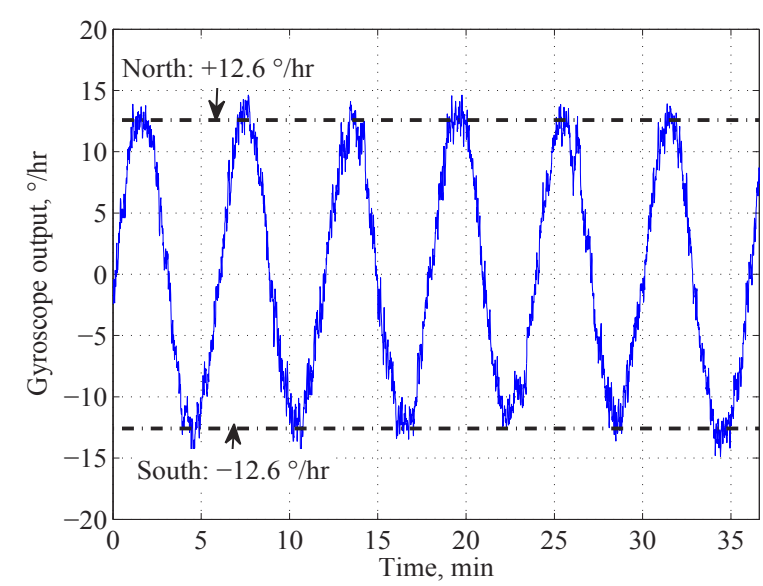

Figure 9: QMG output produced by the Earth's rotation $\left(12.6 \% \mathrm{hr}\right.$ at $33^{\circ}$ latitude) during a $1 \%$ sarouseling, generating amplitude modulation with a 6 min period.

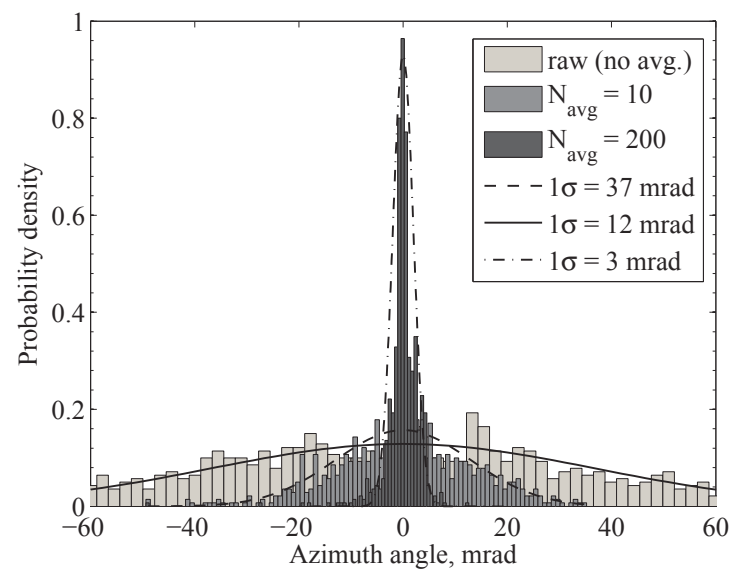

Figure 10: Histogram of 400 azimuth datapoints with normal distribution fits obtained by the carouseling, showing convergence of uncertainty from 37 to $3 \mathrm{mrad}$.

\section{$12.6^{\circ} / \mathrm{hr}$, at North, and minimum, $-12.6^{\circ} / \mathrm{hr}$, at South.}

The carouseling precision was measured by statistical analysis. Every 6 min azimuth was extracted in real-time from a sinusoidal fit. Specifically, azimuth was calculated by taking the difference between the phase of a sinusoidal fit and the instantaneous position of the rate table. Figure 10 shows histogram of 400 raw and averaged azimuth angles. The carouseling approach proved to be robust to scale-factor and bias variations (despite the sensitivity of $-180 \% \mathrm{hr} /{ }^{\circ} \mathrm{C}$ ), thus yielding normal distribution of errors. This allowed us to reduce uncertainty by the data averaging. Normal distribution fits in Figure 10 demonstrate convergence of standard deviation as a number of averages. Similar to maytagging, the uncertainty scaled down as $40 \mathrm{mrad} / \sqrt{ } N$. A $4 \mathrm{mrad}$ uncertainty was reached by filtering of $N=100$ points.

\section{CONCLUSIONS}

We demonstrated a silicon MEMS quadruple mass gyroscope with $0.1 \%$ hr bias instability and 1.2 million $Q$ factor, capable of north-finding with $4 \mathrm{mrad}$ precision. Carouseling and maytagging methods were implemented for true North detection using the Earth's rotation. Both methods produced an azimuth estimation with uncertainty $\sigma$ diminishing as the square root of the number $N$ of turns, $\sigma(N)=40 \mathrm{mrad} / \sqrt{ } N$. The carouseling was robust to bias

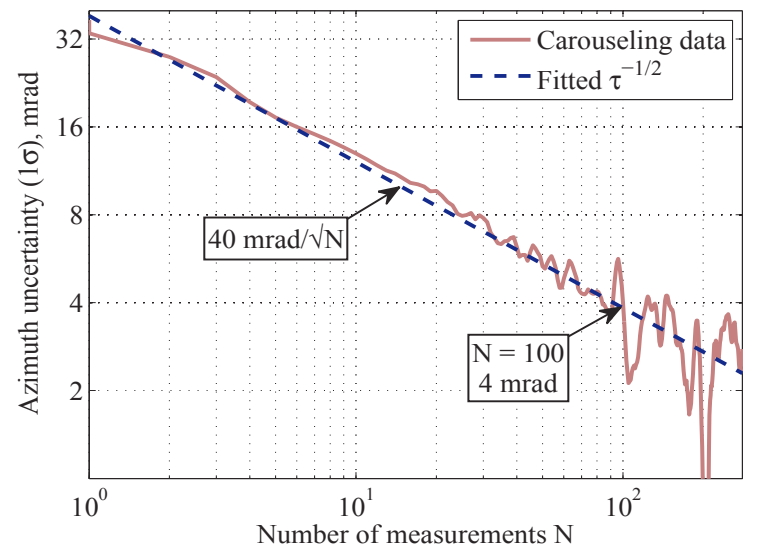

Figure 11: Azimuth uncertainty as a function of number of filtered measurements from Fig. 10 data. A 4 mrad uncertainty is achieved by filtering of $N=100$ points.

and scale factor changes, but required precise continuous rotation. The maytagging relied on $180^{\circ}$ turns, but required temperature calibration. These results clearly show feasibility of silicon inertial MEMS for precision north-finding, currently limited to high resolution macro gyroscopes or magnetic compasses. The ongoing work is expected to reduce north-finding time down to a minute.

\section{ACKNOWLEDGEMENTS}

This work was supported by the ONR/NSWCDD Grants N00014-09-1-0424 and N00014-11-1-0483. The authors thank Dr. Sergei Zotov, Ilya Chepurko, and Dr. Flavio Heer for assistance with the electronics.

\section{REFERENCES}

[1] F. Ayazi, "Multi-DOF inertial MEMS: From gaming to dead reckoning," Proc. Transducers'11 Conf., Beijing, China, June 5-9, 2011, pp. 2805-2808.

[2] B.R. Johnson et al., "Development of a MEMS Gyroscope for Northfinding Applications," Proc. ION PLANS'10, Indian Wells, CA, 2010, pp. 168-170.

[3] I. Prikhodko, S. Zotov, A. Trusov, A. Shkel, "Subdegree-per-hour silicon MEMS rate sensor with 1 million Q-factor," Proc. Transducers'11 Conf., Beijing, China, June 5-9, 2011, pp. 2809-2812.

[4] R. Arnaudov and Y. Angelov, "Earth rotation measurement with micromechanical yaw-rate gyro," IOP Meas. Sci. Technol., vol. 16, pp. 2300-2306, 2005.

[5] A. Trusov, I. Prikhodko, S. Zotov, A. Shkel, "LowDissipation Silicon Tuning Fork Gyroscopes for Rate and Whole Angle Measurements, "IEEE Sensors Journal, vol. 11, no. 11, pp. 2763-2770, 2011.

[6] J.Vig, "Dual-mode oscillators for clocks and sensors," Proc. IEEE Ultrasonics Symp, 1999, pp.859-868.

[7] M. Weinberg, A. Kourepenis, "Error sources in inplane silicon tuning-fork MEMS gyroscopes," $J$. Microelectromech. Syst, vol. 15, pp. 479-491, 2006.

[8] C.M. Jha et al., "CMOS-Compatible Dual-Resonator MEMS Temperature Sensor with Milli-Degree Accuracy," Proc. Transducers'07, 2007, pp. 229-232.

\section{CONTACT}

A.A Trusov, tel: +1-949-8246314; atrusov@uci.edu. 